\title{
Bonding Mechanism from the Impact of Thermally Sprayed Solid Particles
}

\author{
S. GU and S. KAMNIS
}

Power particles are mainly in solid state prior to impact on substrates from high velocity oxyfuel (HVOF) thermal spraying. The bonding between particles and substrates is critical to ensure the quality of coating. Finite element analysis (FEA) models are developed to simulate the impingement process of solid particle impact on substrates. This numerical study examines the bonding mechanism between particles and substrates and establishes the critical particle impact parameters for bonding. Considering the morphology of particles, the shear-instabilitybased method is applied to all the particles, and the energy-based method is employed only for spherical particles. The particles are given the properties of widely used WC-Co powder for HVOF thermally sprayed coatings. The numerical results confirm that in the HVOF process, the kinetic energy of the particle prior to impact plays the most dominant role in particle stress localization and melting of the interfacial contact region. The critical impact parameters, such as particle velocity and temperature, are shown to be affected by the shape of particles, while higher impact velocity is required for highly nonspherical powder.

DOI: $10.1007 / \mathrm{s} 11661-009-9959-1$

(C) The Minerals, Metals \& Materials Society and ASM International 2009

\section{INTRODUCTION}

HiGH velocity oxy-fuel (HVOF) thermal spraying has been applied successfully in producing coating with higher density, superior bond strengths, and less decarburization due to its unique advantage of high momentum and low thermal output. ${ }^{[1-5]}$ The experimental measurements of in-flight particles ${ }^{[6,7]}$ show low-temperature profiles for HVOF-sprayed powder, which enables powder particles with a high melting point to remain in their solid state prior to impact, especially in liquid fueled HVOF systems. Such behavior is further confirmed by the simulation of HVOF-sprayed in-flight particles. ${ }^{[8-10]}$ The impingement of liquid droplets including spreading, breakup, air entrapment, and solidification has been studied in References 11 and 12, while the solid particle impact and its subsequent bonding mechanism has not been well understood. Considering a normal deposition efficiency of $\sim 50 \mathrm{pct}$ for HVOF coating, it is important to have a good understanding of the intricate interaction between kinetic and thermal energy of particles and the bonding mechanism of coating, for effective control of the process. However, such quantitative analyses of HVOF coating have not been reported. The closest information is for the study of cold spraying, which gives more kinetic energy and a small amount of heat to the sprayed particles. Experimental observation ${ }^{[13-16]}$ and numerical simulation ${ }^{[17-19]}$ of the solid particle

S. GU, Senior Lecturer, and S. KAMNIS, Research Fellow, are with the School of Engineering Science, University of Southampton, Highfield, Southampton SO17 1BJ, United Kingdom. Contact e-mail: s.gu@soton.ac.uk

Manuscript submitted January 16, 2009.

Article published online August 22, 2009 deformation in cold spraying shows that bonding is the result of extensive plastic deformation and related phenomena at the impact interface. Quantitative analyses ${ }^{[17-19]}$ of the relationship between the deposition efficiencies and particle impact velocities indicate a critical particle velocity for successful bonding. Below the critical velocity, solid particles rebound from the substrate, which causes densification and abrasion similar to the shot pinning method. Above this critical velocity, particles deform plastically and bond with the substrate. Thermal softening due to plastic deformation needs to overcome the strain-hardening effect resulting in thermal-plastic shear instability, ${ }^{[20,21]}$ which takes place in a thin region at the contact interface and is attributed to bonding. ${ }^{[19]}$ The presence of both high kinetic energy and substantial thermal energy from HVOF-sprayed particles gives added complexity to quantify the critical impact parameter for successful bonding. The complexity means both particle velocity and temperature from HVOF spraying influence the bonding, instead of only particle velocity in the case of cold spray, or mainly particle temperature in arc, plasma, and other thermal spray processes. Thermally sprayed powder particles are varied in shape and size, according to the technology of powder production. For instance, gas-atomized powder has good spherical shape. Hard metals such as WC-Co powders are not spherical when they are made from mechanical milling. The effect of powder morphology on the quality of coating has not been examined extensively.

This article presents a numerical approach to examining the bonding mechanism of HVOF thermal spray coating and to establishing the critical impact velocities of bonding within a wide range of parameters. The popular hard materials, WC-Co powders, are selected in this study, and the particle parameters prior to impact 
are taken from the previous computational fluid dynamics (CFD) in-flight particle models. ${ }^{[8,9]}$

\section{MODEL DEVELOPMENT}

Solid impact dynamics are analyzed by using the finite element commercial solver ABAQUS/Explicit (Dassault Systèmes, Suresnes, France). The model accounts for strain hardening, thermal softening, and heating due to frictional and plastic dissipation. Due to very small particle size and time scales (nanoseconds) related to the HVOF particle impact process, heat transfer between particles and substrates can be neglected, so heating is assumed to be adiabatic. ${ }^{[17,19]}$ The validity of this adiabatic assumption can be assessed by the dimensionless parameter $\frac{x^{2}}{D \cdot t}$, where $x$ is a characteristic system dimension, $D$ is thermal diffusivity, and $t$ is the process time. It can be considered as adiabatic heating when $\frac{x^{2}}{D \cdot t} \geq 1$. Given $x=10^{-5} \mathrm{~m}, \quad D=10^{-6} \mathrm{~m}^{2} / \mathrm{s}$, and $t=10 \mathrm{~ns}, \frac{x^{2}}{D \cdot t}$ is well above unity in a typical case of this simulation. The preceding discussion is based on the solution of the diffusive heat equation. At a very small scale, heat conduction is expected to be dominated by a wave propagation mechanism rather than by diffusion. This implies that with the small particle size, the heat propagation would approach the speed of plastic waves and be limited by the speed of sound in the particle. In other words, heat conduction could be even slower than the prediction from the diffusive heat equation for the high speed impact of very small particles. A good description of the preceding argument can be found in References 22 through 24.

The material properties are summarized in Table I, and the powder composition can be found in Reference 25 . The elastic response of the material follows a linear elasticity model, which is adequate for most impact cases. The plastic response of WC-Co is assumed to comply with the widely used Johnson-Cook plasticity model $^{[26]}$ as follows:

$$
\begin{gathered}
\tau=\left(\mathrm{A}+\mathrm{B} \bar{\gamma}_{p}^{n}\right) \cdot\left(1+C \ln \frac{\dot{\gamma}}{\dot{\gamma}_{0}}\right) \cdot\left[1-\left(\frac{T-T_{0}}{T_{m}-T_{0}}\right)^{m}\right] \\
T=T_{0}+\frac{\beta}{\rho c_{p}} \int \tau d \bar{\gamma}_{p}
\end{gathered}
$$

Table I. Material Properties of WC-17Co Powder ${ }^{[29]}$

\begin{tabular}{ll}
\hline Density (WC-17Co) $\left(\mathrm{kg} / \mathrm{m}^{3}\right)$ & 14,000 \\
Solidus temperature $(\mathrm{Co})(\mathrm{K})$ & 1580 \\
Liquidus temperature $(\mathrm{Co})(\mathrm{K})$ & 1640 \\
Specific heat (WC-17Co) $(\mathrm{J} / \mathrm{kg} \mathrm{K})$ & 295 \\
Latent heat (WC-17Co) $(\mathrm{J} / \mathrm{kg})$ & 420,000 \\
Young's modulus (WC-17Co) (GPa) & 500 \\
Poisson's ratio (WC-17Co) & 0.27 \\
Shear strength (WC-17Co) (MPa) & 95 \\
Static shear strength (WC-17Co) (GPa) & 1.55 \\
Strain-hardening modulus (WC-17Co) $(\mathrm{GPa})$ & 22 \\
Strain-rate-sensitive coefficient $(\mathrm{WC}-17 \mathrm{Co})$ & 0.0312 \\
Thermal-softening exponent (WC-17Co) & 1.34 \\
Strain-hardening exponent (WC-17Co) & 0.45 \\
\hline
\end{tabular}

where $\bar{\gamma}_{p}$ is the average plastic shear strain; $\dot{\gamma}_{0}$ is the reference shear strain rate; $\dot{\gamma}$ is the imposed shear strain rate; $\tau$ is the flow stress; $T$ is the particle temperature; $T_{0}$ is the impact temperature; $T_{m}$ is the melting temperature; $\beta$ is the work to heat conversion factor (based on the empirical assumption that 90 pet of the kinetic energy is dissipated to heat allowing for heat conduction within the particle); $c_{p}$ is the heat capacity; $\rho$ is the density; and $A, B, C, m$, and $n$ are material-dependent constants such as static shear strength, strain-hardening modulus, strain-rate-sensitive coefficient, thermal-softening exponent, and strain-hardening exponent, respectively.

For the energy-based calculations, the energy required $^{[27,28]}$ for bouncing the particle from the substrate is expressed as follows:

$$
E_{R}=\frac{1}{2} c_{r} m_{p} u_{p}^{2}
$$

where $m_{p}$ and $u_{p}$ are the mass and velocity of the particle. The expression of the recoil coefficient $c_{r}$ can be found in Reference 29 and is not shown here for brevity. The strain-hardening, thermal-softening, and deformation localization are considered for the calculation of the recoil coefficient and are provided from the Johnson-Cook plasticity model. The energy required to detach a bonded particle from the substrate is expressed as follows: ${ }^{[30]}$

$$
E_{A}=a \text { pct } A_{\max }
$$

where $A_{\max }$ is the maximum adhesion energy; and $a$ pct is the relative strength of the bond between the particle and substrate and is mainly affected by the particle velocity and contact temperature. Detailed expressions for maximum adhesion energy and relative bond strengths can be found in Reference 29 and are not repeated here.

For nonspherical particles, the degree of sphericity has to be taken into account. This is quantified by the shape factor:

$$
S F=\frac{A_{s p}}{A}
$$

where $A_{s p}$ is the surface area of the equivalent sphere at the same volume as the original particle, and $A$ is the actual surface area of the original particle. The surface area of the equivalent sphere is

$$
A_{s p}=\pi^{1 / 3}(6 V)^{2 / 3}
$$

where $V$ is the particle volume. The corresponding diameter of the equivalent sphere is

$$
D_{s p}=\left(\frac{6 V}{\pi}\right)^{1 / 3}
$$

The shape factors for selected configurations used in this study are given in Table II with increasing sharpness as circular, orthogonal, hexagonal, and quadrangular.

The computational domain for the impingement process is shown in Figure 1. The substrate dimension (300 $\mu \mathrm{m}$ in length and width, and $200 \mu \mathrm{m}$ in height) is given to be $\sim 5$ times larger than the particle diameter 
Table II. Shape Factors of Particles Used in the Study

Shape Factor

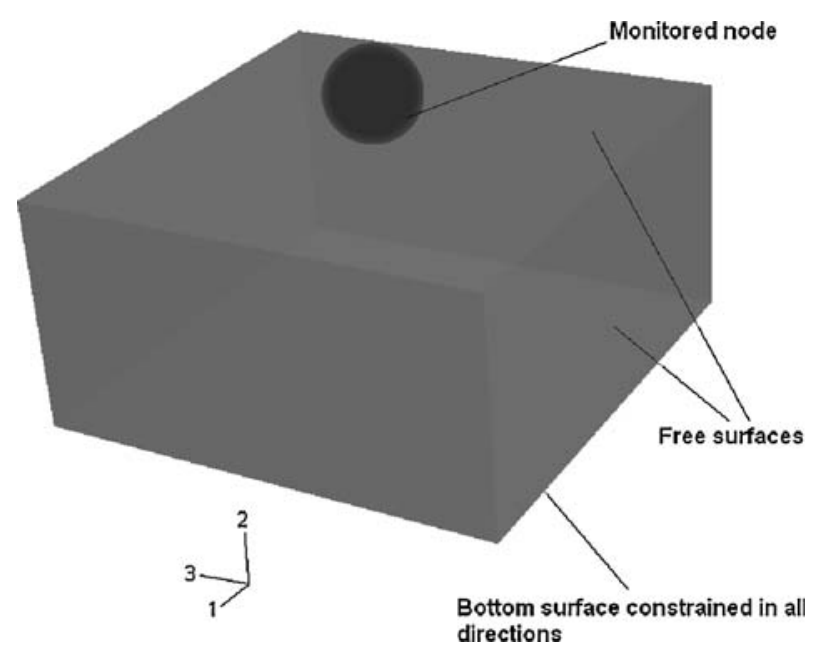

Fig. 1-Computational domain for 3-D finite element model.

(the largest $60-\mu \mathrm{m}$ particle) to avoid possible effects on the particle-substrate deforming zones from the boundary nodes. The substrate bottom face is constrained in all directions, while the other faces are set as free. Reports on grid sensitivity have shown that the mesh size can play a dominant role in material heating and, consequently, in shear flow localization. ${ }^{[19]}$ For this reason, very fine meshes are employed for both particle and substrate. For instance, $40-\mu \mathrm{m}$ spherical particle has a mesh size of $0.5 \mu \mathrm{m}$ corresponding to approximately 700,000 four-node linear tetrahedron elements. The results show that maximum temperature at the interface is very sensitive to the element size. To eliminate such grid effect, the maximum temperature at the interface is calculated by linear extrapolation to "zero element size" proposed by Reference 19. In this model, the mesh size is varied from 1 to $0.1 \mu \mathrm{m}$, until the "predetermined" maximum temperature is reached. The velocity and temperature of spray particles prior to impact on the substrate are taken from the CFD in-flight particle models ${ }^{[8]}$ according to the particle size and shape. A monitor node is selected on the surface of the impacting particle at a location where intensive plastic deformation is expected.

\section{RESULTS AND DISCUSSION}

As a first step in a series of work to examine the bonding mechanism of thermally sprayed coating, conditions simulated in this work cannot be considered as a full representative of a real application. However, the results can be regarded as a baseline for understanding the influence of kinetic energy and thermal phenomena on coating formation. It is known that, prior to thermal spraying, substrate surfaces are prepared by blasting to enhance bonding. An accurate representation of the real application should have the detailed surface profiles of the substrate, which will require extensive computation with sophisticated geometry and grid. In this initial work, the substrate is defined as flat surface with the same property as the WC-Co powder, which can be regarded as the preformed base coat. The substrate has a uniform temperature of $300 \mathrm{~K}$. The particle parameters are varied in terms of particle diameter, shape, impact velocity, temperature, and orientation. The $40-\mu \mathrm{m}$ spherical particles are selected as the baseline particles in this study.

In principle, pure material is more appropriate for this model. WC-Co is considered because it is a very popular material for thermal spray coating; moreover, modeling of this material has not been extensively covered in the literature. To make the model work, some assumptions are given here. We will work to replace them with more accurate approaches in the future. The particle is assumed to melt when the Co element reaches the liquidus temperature of $1640 \mathrm{~K}$. Melting is assumed to take place in a thin interfacial region between the particle and substrate. The remained solid particle will deform according to Eqs. [1] and [2]. It is believed that the viscous-type deformation is only limited to the very thin region where mechanical interlocking takes place to enable bonding. The assumption of $\mathrm{WC} / \mathrm{Co}$ in one state within this thin layer should not make a substantial difference to the overall impact dynamics.

\section{A. Spherical Particle Impingement}

The temperature contours from sprayed spherical particles having a temperature of $800 \mathrm{~K}$ and landing at 300 and $500 \mathrm{~m} / \mathrm{s}$, respectively, are presented in Figure 2 for selected time after impact. The particle starts to deform immediately after impact, causing the formation of a crater on the substrate. In the early stage of impingement (30 ns), the deformation of the contact surface is evident and the crater size increases in width and height to accommodate the deformed particle. At $130 \mathrm{~ns}$, when the particle kinetic energy has fallen to zero, the particle flattens to a lenslike shape. The particle deforms within the contact zone, where the maximum plastic strain is found at numerous locations. At the localized contact interface, a significant temperature increase is observed, as a result of kinetic energy being converted to internal energy and part of the internal energy to plastic work (dissipated as heat). In this region, the temperature rises to $1171 \mathrm{~K}$ at $130 \mathrm{~ns}$. It should be noted that the particle/substrate interface (contact surface) never reaches the melting point of $1640 \mathrm{~K}$ under the impact velocity of $300 \mathrm{~m} / \mathrm{s}$. The contact surface temperatures at $130 \mathrm{~ns}$ are shown in Figure 3, where the interfacial region for the particle is on the top and the substrate at the bottom. The results 
Particle size: $40 \mu \mathrm{m}$

Temperature at impact: $800 \mathrm{~K}$

Impact Velocity: $300 \mathrm{~m} / \mathrm{s}$
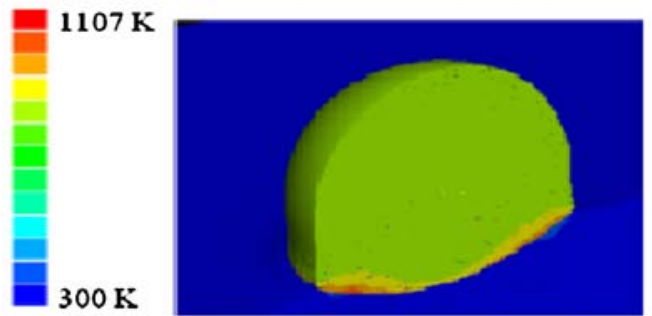

$30 \mathrm{~ns}$
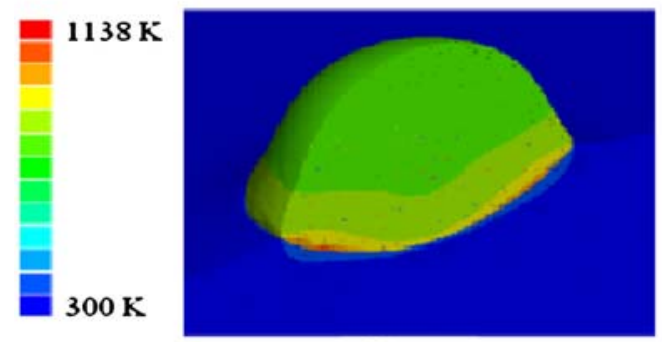

$50 \mathrm{~ns}$
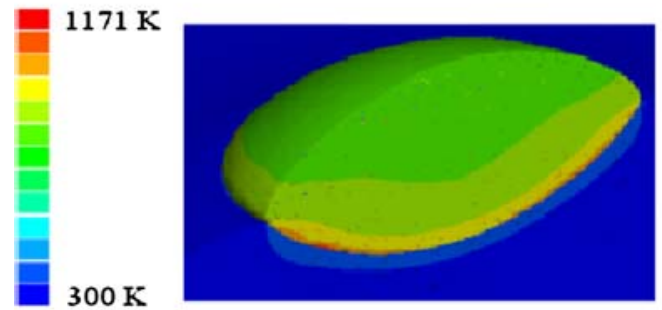

$130 \mathrm{~ns}$
Impact Velocity: $500 \mathrm{~m} / \mathrm{s}$
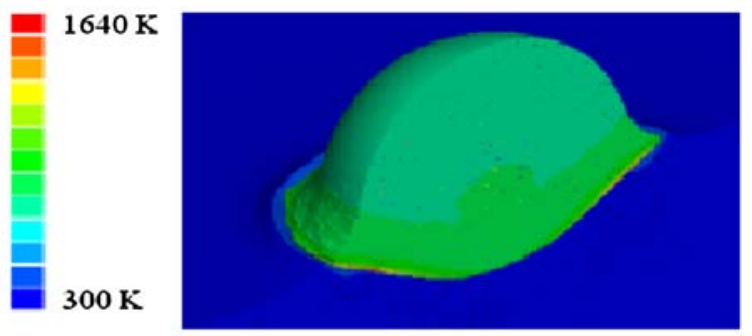

$30 \mathrm{~ns}$

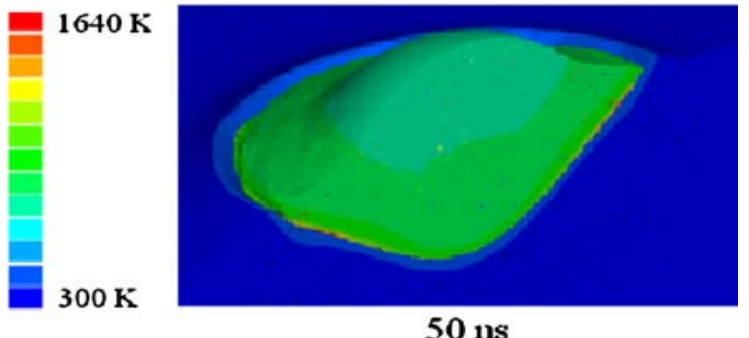

$1640 \mathrm{~K}$

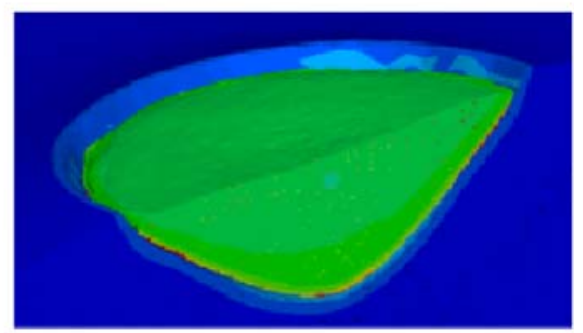

$130 \mathrm{~ns}$

Fig. 2-Particle temperature contours for $40-\mu \mathrm{m}$ particle with impact temperature of $800 \mathrm{~K}$.

show that the temperature is not uniformly distributed over the surfaces due to different plastic strain rates. A higher temperature is observed along the highly deformed zones, around the edge of the particle in comparison to its center. The temperature contours on the substrate surface indicate that the substrate temperature increases much less than the particle, which is attributed to the smaller plastic deformation of the substrate.

A significant difference is observed when the particle impact velocity is increased from 300 to $500 \mathrm{~m} / \mathrm{s}$. On the right side of Figure 2, the particle penetrates deeper into the substrate, while the edges of the particle are pushed outward, forming a jetlike shape at the periphery of the contact zone due to the liquid instability and solidification at the edges. The particle is plastically deformed to a greater extent and the temperature at the interface reaches the melting point $(1640 \mathrm{~K})$. The melting of the particle at $500 \mathrm{~m} / \mathrm{s}$ is more pronounced, as shown in Figure 3, where a much larger molten area around the particle edge is clearly seen at $130 \mathrm{~ns}$. Within this molten area, the deformation mechanism of the particle has changed from plastic to viscous flow. The extensive thermal softening in this region results in a low resistance to shear flow. Near the melting point, the material loses its shear strength and undergoes excessive deformation. The result of such viscous-type flow can be explained by Raleigh-Taylor instabilities, ${ }^{[11]}$ which in turn promote adhesion through mutually conforming contacting surfaces. Due to these softening effects, the particle is deformed to a greater extent than the substrate, resulting in lower crater surface temperature, as shown in Figure 3 (bottom right). The temperature on the substrate interface is also increased to $556 \mathrm{~K}$. A similar phenomena of particle deformation and melting from high-speed particles have been reported in cold spraying. ${ }^{[13-16]}$ Experimental observations on the surface and cross section of cold-sprayed $\mathrm{Cu}$ particles clearly show a lenslike shape of the splats, jetting at the interfacial regions (indicating of melting) and creating a crater in the substrate, ${ }^{[13-16]}$ which is in good agreement with the simulated results.

\section{B. Effect of Particle Impact Velocity}

Section A described the overall impact dynamic, and this section focuses on the results of the monitored node. In this study, the particle remains at the impact 
Particle size: $40 \mu \mathrm{m}$

Temperature at impact: $800 \mathrm{~K}$
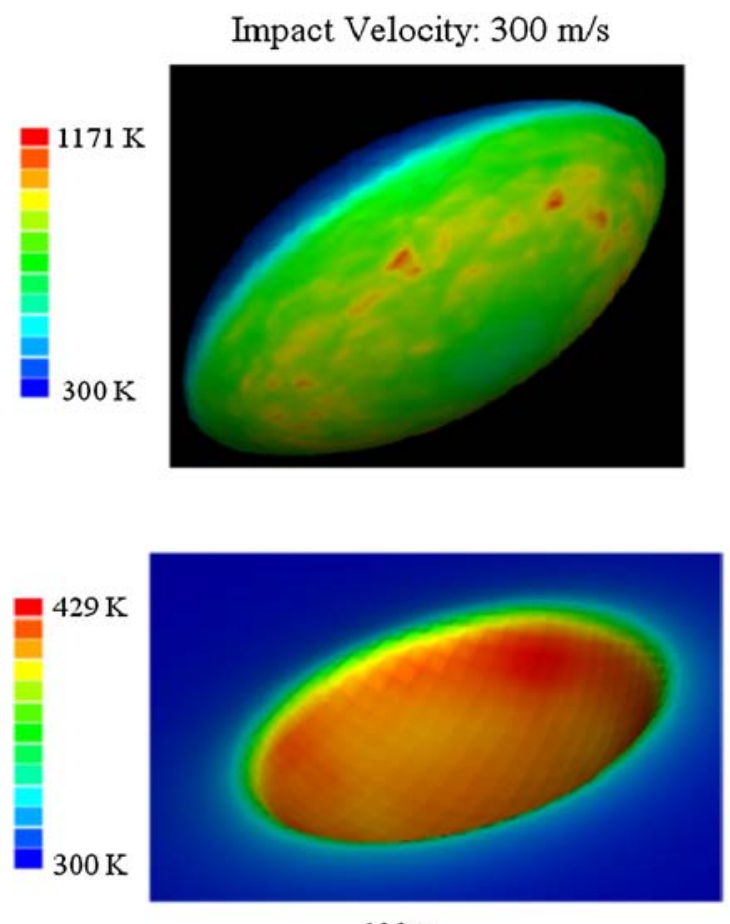

$130 \mathrm{~ns}$
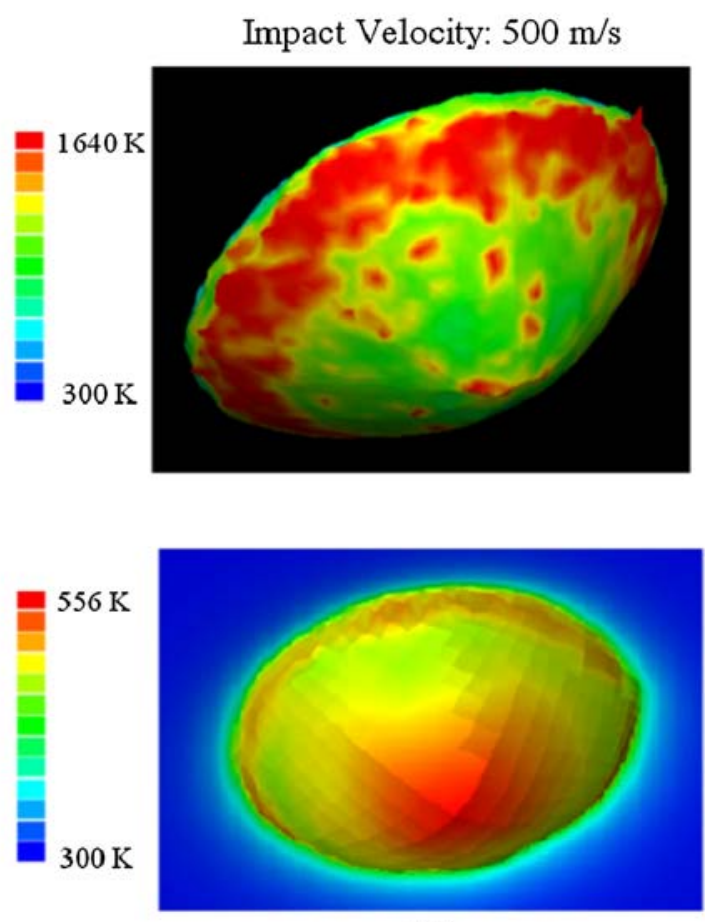

$130 \mathrm{~ns}$

Fig. 3-Interfacial temperature contours for $40-\mu \mathrm{m}$ particle with impact temperature of $800 \mathrm{~K}$ particle at $130 \mathrm{~ns}$ (top: particle contact surface; and bottom: substrate crater).

temperature of $800 \mathrm{~K}$, while the impact velocities are varied at 300,400 , and $500 \mathrm{~m} / \mathrm{s}$. The temperature and flow stress evolution on the monitored node, which undergoes the highest amount of deformation within the particle, are shown separately in Figures 4 and 5. The temporal development of temperature in the monitored node for velocities smaller than $500 \mathrm{~m} / \mathrm{s}$ follows the same trend as that of flow stress. The temperature reaches the melting point at the impact velocity of $500 \mathrm{~m} / \mathrm{s}$, whereas in other cases, it remains well below the melting point. An increase of $100 \mathrm{~m} / \mathrm{s}$ in the impact velocity results in a temperature increase of almost $400 \mathrm{~K}$ in the monitored node. For impact velocities smaller than $500 \mathrm{~m} / \mathrm{s}$, the stress profiles increase with the impact velocity, which is attributed to the loading conditions (the compressive load on the particle) and thermal-softening effect. A significant change in stress development occurs when the impact velocity reaches $500 \mathrm{~m} / \mathrm{s}$. As the melting temperature is reached, the variation of stress is characterized by instabilities and a decrease in the overall magnitude close to zero. This drop can be explained with respect to the change of deformation mechanism from plastic to viscous. Under such conditions, the shearing and heating becomes highly localized, while the straining and heating practically stops. From the temperature plot in Figure 4, no further heating of the material is observed under stress localization at $500 \mathrm{~m} / \mathrm{s}$. Consequently, more effective bonding of the particle on the substrate is achieved

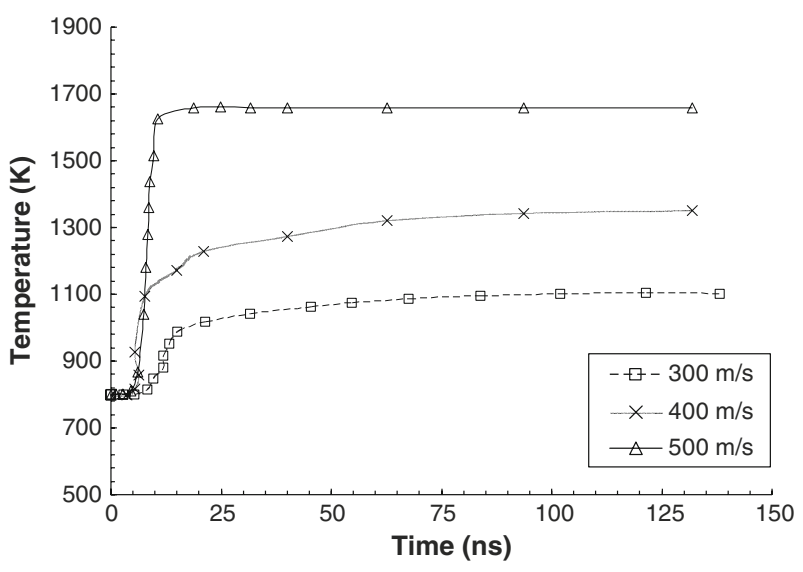

Fig. 4-Temperature evolutions at the monitored point with different impact velocities.

under such conditions, as pointed out in the previous cold spray model. ${ }^{[19]}$

\section{Effect of Particle Impact Temperature}

Unlike cold spray, in the HVOF process, the particles are accelerated through momentum transfer from hot combusting gases. Heat is also transferred to the particles from the surrounding gas flow. It remains an open question whether the kinetic or the thermal energy is the 


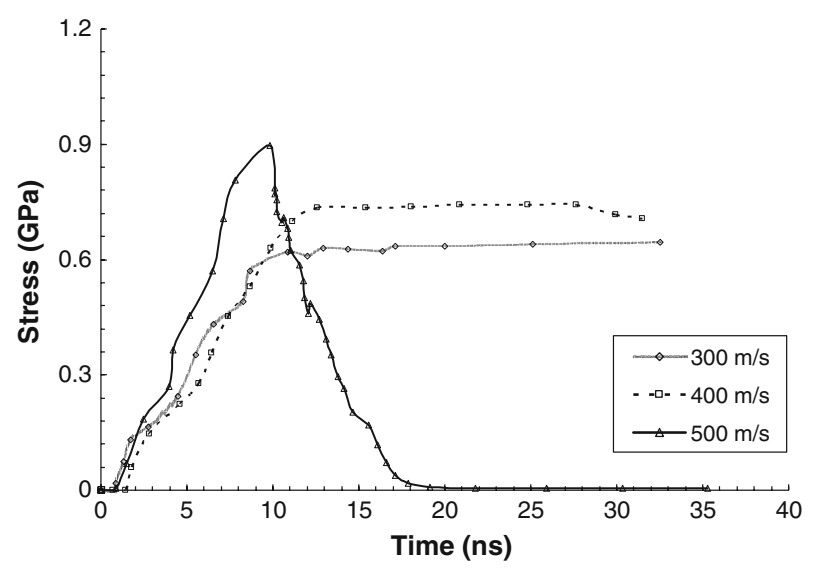

Fig. 5-Flow stress evolutions at the monitored point with different impact velocities.

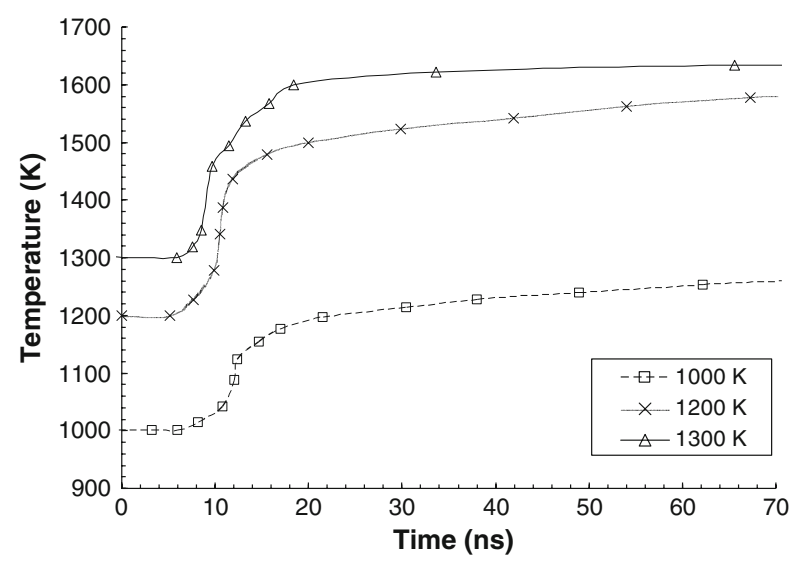

Fig. 6-Temperature evolutions at the monitored point with different impact temperatures.

driving force for successful particle bonding. To address this issue, different particle impact temperatures, namely, 1000,1200 , and $1300 \mathrm{~K}$, are used with the same impact velocity of $300 \mathrm{~m} / \mathrm{s}$. The effect of initial temperature on the thermal and stress behaviors of the particle upon impact is shown in Figures 6 and 7. The evolution of temperature and flow stresses follows the same pattern as described previously when the impact velocity is increased gradually. The increase of impact temperature with fixed impact velocity results in a very similar increase of monitored node temperature. The temperature at the monitored point increases by $300 \mathrm{~K}$ by changing the impact temperature from 1000 to $1300 \mathrm{~K}$, whereas an increase of $\sim 600 \mathrm{~K}$ is found by changing the impact velocity from 300 to $500 \mathrm{~m} / \mathrm{s}$. These results indicate that the plastic work being dissipated to heat is not significantly altered at different particle impact temperatures. The kinetic energy being converted to heat in these cases remains the same, and the difference in final monitored node temperature is attributed to the impact temperature plus a constant heat for all cases from plastic work dissipation. The comparison clearly demonstrates that the kinetic energy of the particle prior to impact plays the most important role in particle stress localiza-

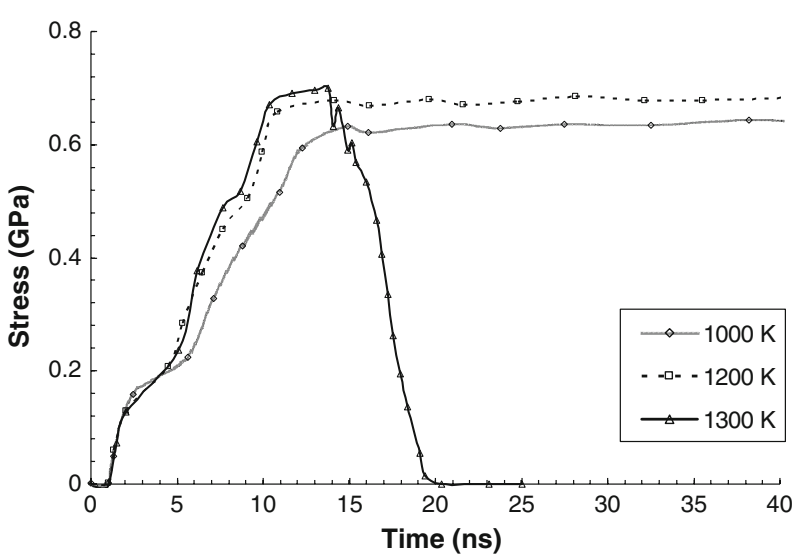

Fig. 7-Flow stress evolutions at the monitored point with different impact temperatures.

tion and melting of the interfacial region. Practically, this means that to improve the quality of HVOF coatings, more attention should be paid to increasing the momentum output rather than the thermal output.

\section{Effect of Particle Morphology}

Gas atomization produces spherical powder, while the milling process generates nonspherical particles. The change of particle shape will affect both the in-flight particle dynamics and the impingement process. Table III shows the particle impact parameters calculated from the CFD in-flight particle models for the HVOF process. ${ }^{[8]}$ It is shown that nonspherical particles reach higher average velocities than the spherical powder at the same operation parameters due to higher drag coefficient for nonspherical particles. From the preceding analysis of spherical particles, it is clear that the kinetic energy prior to impact is a key factor for strong adhesion. Based purely on the value of impact velocity, the nonspherical powder should have generated a better coating from the HVOF process. In fact, particle morphology also influences the deformation rates during particle impingement. To examine such an effect, the impingements from four $40-\mu \mathrm{m}$ particles with different morphologies are compared in Figure 8. The particles are given the same critical impact parameters for the spherical particle, i.e., impact velocity of $420 \mathrm{~m} / \mathrm{s}$ and impact temperature of $750 \mathrm{~K}$. The results show that all the particles create a crater on the substrate. The interfacial region between the particle and substrate reaches a lower temperature for particles with a lower shape factor. The resistance to the temperature increase in the interfacial region is caused by the lower levels of plastic deformation at low SF. The decreased plastic deformation is attributed to the increased contacting area between the nonspherical particles and the substrate at which impact subsequently reduces flow stress concentration compared to the spherical particle. The results demonstrate that higher impact velocities are required for highly nonspherical particles such as SF 0.8 to achieve particle melting at the interfacial region, which enables the formation of bonding required for mechanical interlocking of the particle substrate. 
Table III. Computational Results for Particle Velocity and Temperature at Impact

\begin{tabular}{|c|c|c|c|c|c|c|c|c|}
\hline \multirow[b]{2}{*}{ Particle Diameter $(\mu \mathrm{m})$} & \multicolumn{2}{|c|}{ SF 1} & \multicolumn{2}{|c|}{ SF 0.95} & \multicolumn{2}{|c|}{ SF 0.9} & \multicolumn{2}{|c|}{ SF 0.8} \\
\hline & $\mathrm{V}(\mathrm{m} / \mathrm{s})$ & $T(\mathrm{~K})$ & $\mathrm{V}(\mathrm{m} / \mathrm{s})$ & $T(\mathrm{~K})$ & $\mathrm{V}(\mathrm{m} / \mathrm{s})$ & $T(\mathrm{~K})$ & $\mathrm{V}(\mathrm{m} / \mathrm{s})$ & $T(\mathrm{~K})$ \\
\hline $20 \mu \mathrm{m}$ & 566 & 1005 & 560 & 1050 & 575 & 1100 & 655 & 972 \\
\hline $40 \mu \mathrm{m}$ & 420 & 751 & 425 & 740 & 435 & 732 & 470 & 670 \\
\hline
\end{tabular}

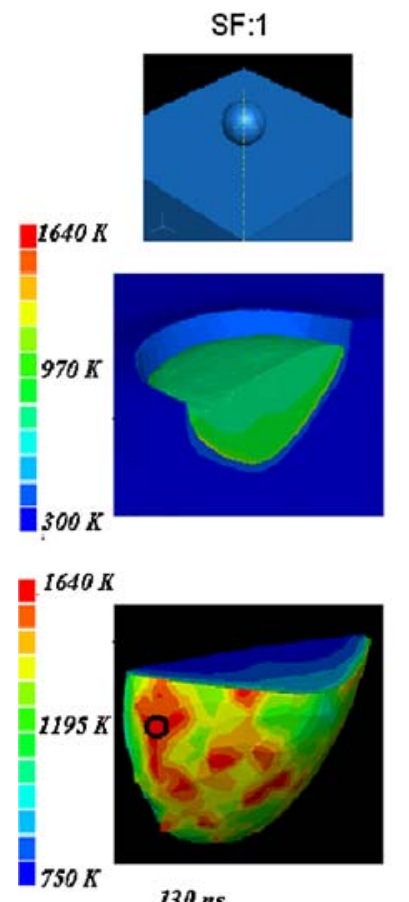

$130 \mathrm{~ns}$
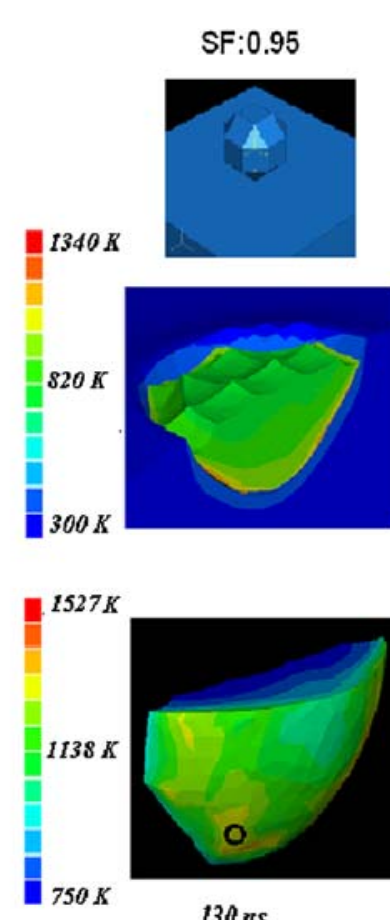
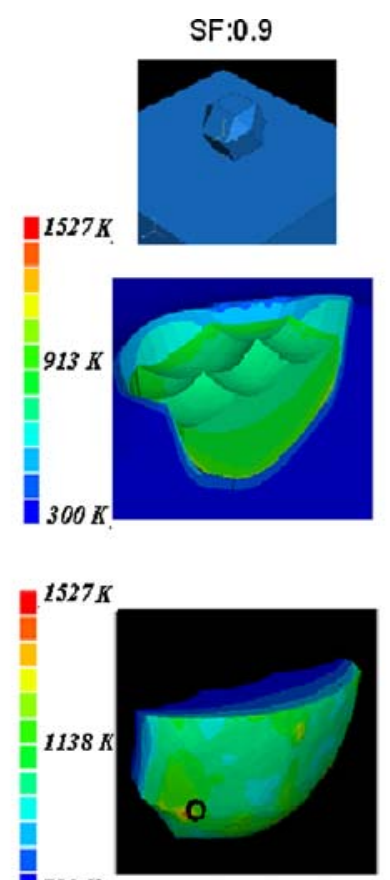

$130 \mathrm{~ns}$
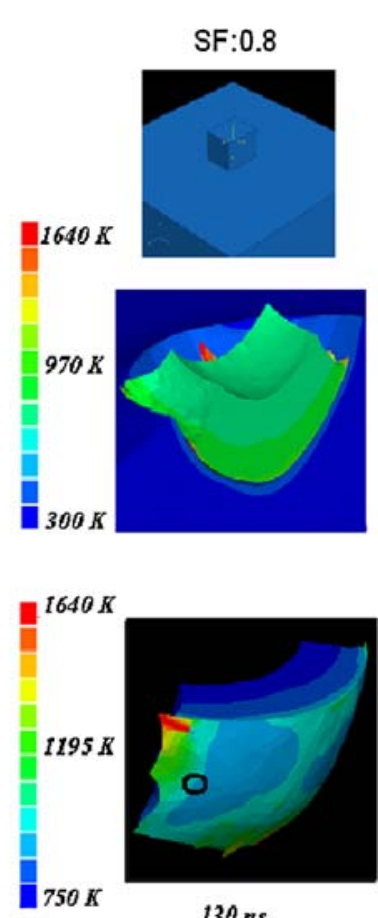

$130 \mathrm{~ns}$

Fig. 8-Temperature contours for different shape $40-\mu \mathrm{m}$ particles with an impact temperature of $750 \mathrm{~K}$ and velocity $420 \mathrm{~m} / \mathrm{s}$ at $130 \mathrm{~ns}$ (top: before impact; middle: after impact; and bottom: interfacial contact surface).

For more quantitative comparison, a point in the maximum deformation area for each particle is selected, as illustrated in the black circles in Figure 8. The temperature developments during the course of impingement at those points are plotted in Figure 9. It is apparent that the temperatures increase during impingement, which results in more strain and deformation. The most substantial rise in temperature occurs at the initial state of impingement $(0$ to $30 \mathrm{~ns}$ ), where the kinetic energy is at the highest level. The temperature increase becomes steady between 30 and 80 ns. From then, a slightly more pronounced temperature rise is visible, particularly for the spherical particle. On impact, the kinetic energy of the powder particle is distributed at a molecular level, resulting in a temperature rise due to work done during plastic deformation and a phase change from solid to liquid. As the stresses propagate through the particle, the temperature rises and a phase change follows. Once the powder particle is entirely in its liquid state, the rate of increasing temperature grows as no more latent energy is being absorbed. Such a temperature increase is less noticeable as the particle becomes less spherical, because the nonuniform surface prohibits an efficient

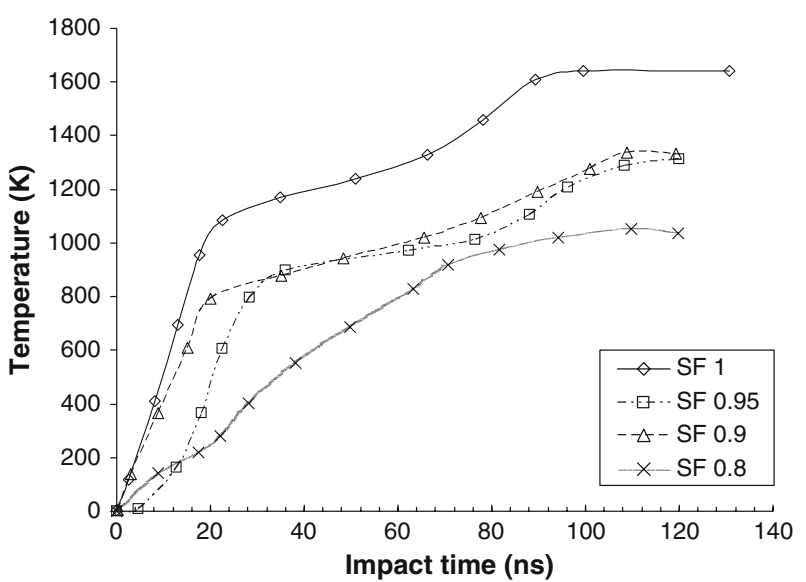

Fig. 9-Temperature developments during the course of impingement at the critical nodes for different shape particles.

distribution of an efficient conversion and distribution of the particle's initial kinetic energy to thermal and latent energy. The microstructure analysis of thermally sprayed coating ${ }^{[31]}$ confirms the simulation results that 
spherical powder gives a denser coating, whereas the milled nonspherical powder does not exhibit the same extent of deformation, leading to a porous coating.

\section{E. Effect of Particle Orientation}

The gas flow in the HVOF spray process is characterized by very high turbulence intensity, particularly in the supersonic jet region where the hot combusting gases are mixed with ambient air. The velocity fluctuation in three-dimensional (3-D) form has a direct influence on the motion of powder particles. In the case of nonspherical powder, the particles are more sensitive to flow fluctuations; therefore, the particle orientation at the impact moment can be different for similar particles at the same operation conditions. It is interesting to know to what extent this impact orientation affects the bonding strength. Figure 10 shows the temperature contours of deformed particles having a shape factor of 0.8 for two different impingement orientations. The particle is impacting with a velocity of $420 \mathrm{~m} / \mathrm{s}$ and a temperature of $750 \mathrm{~K}$. The temperature distributions over the interfacial regions clearly show that the temperature increases, which corresponds to high deformation when the contact area between the particle and
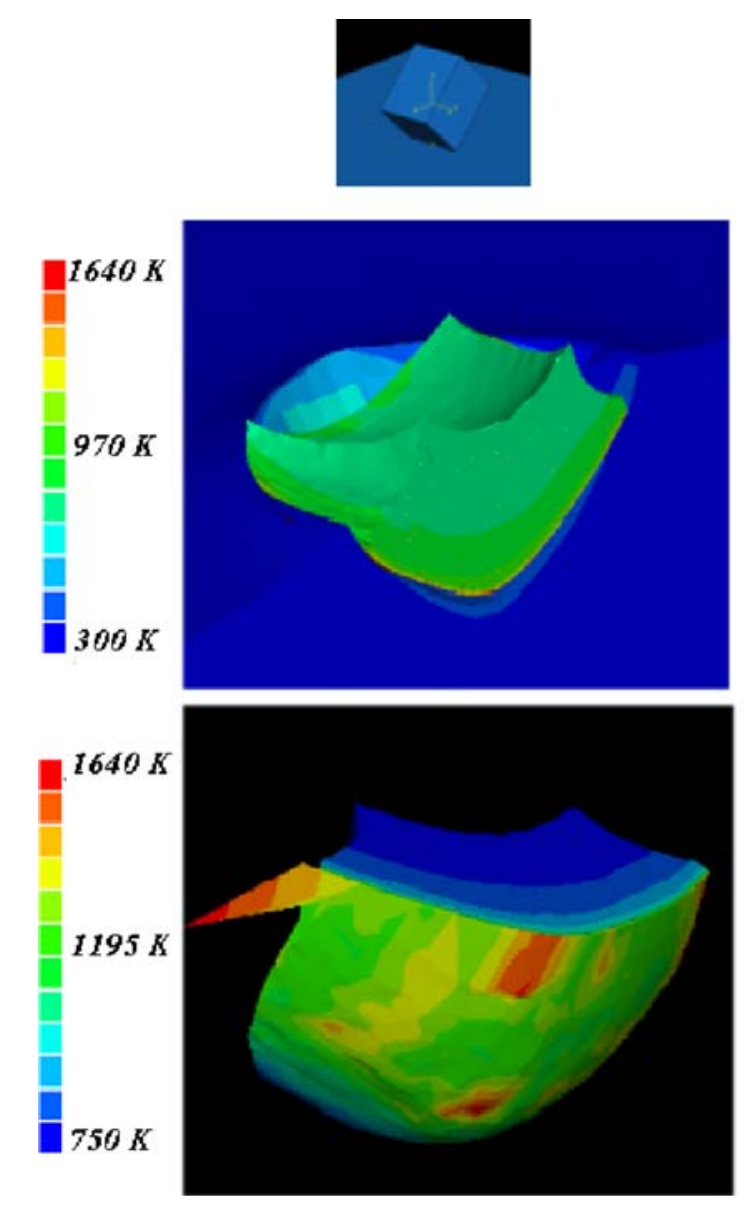

substrate is decreased. The substantial influence of particle orientation at the impact implies another possible means of improving the deposition efficiency of coating, by directing the nonspherical particles toward the desirable orientations.

\section{F. Critical Impact Conditions}

The preceding analysis is based on a $40-\mu \mathrm{m}$ particle size. In reality, the range of particle sizes of thermally sprayed powder is 5 to $60 \mu \mathrm{m}$. It is necessary to know the critical impact velocities and temperatures for different particle sizes and morphologies to obtain adequate bonding strengths. Figure 11 plots the critical velocity as a function of impact temperature for spherical powders with sizes 20,40 , and $60 \mu \mathrm{m}$. The results indicate that a proportional increase of critical velocity is required as the particle becomes larger. The $X$ points represent the particle impact velocities and temperatures obtained from the CFD models for inflight particle dynamics. ${ }^{[8]}$ For the particles located above the lines, the impact temperature and velocity are adequate to ensure adequate bonding with the substrate. For the range of particles used, only powder sizes smaller than $40 \mu \mathrm{m}$ have enough kinetic and thermal energy to result in successful bonding.

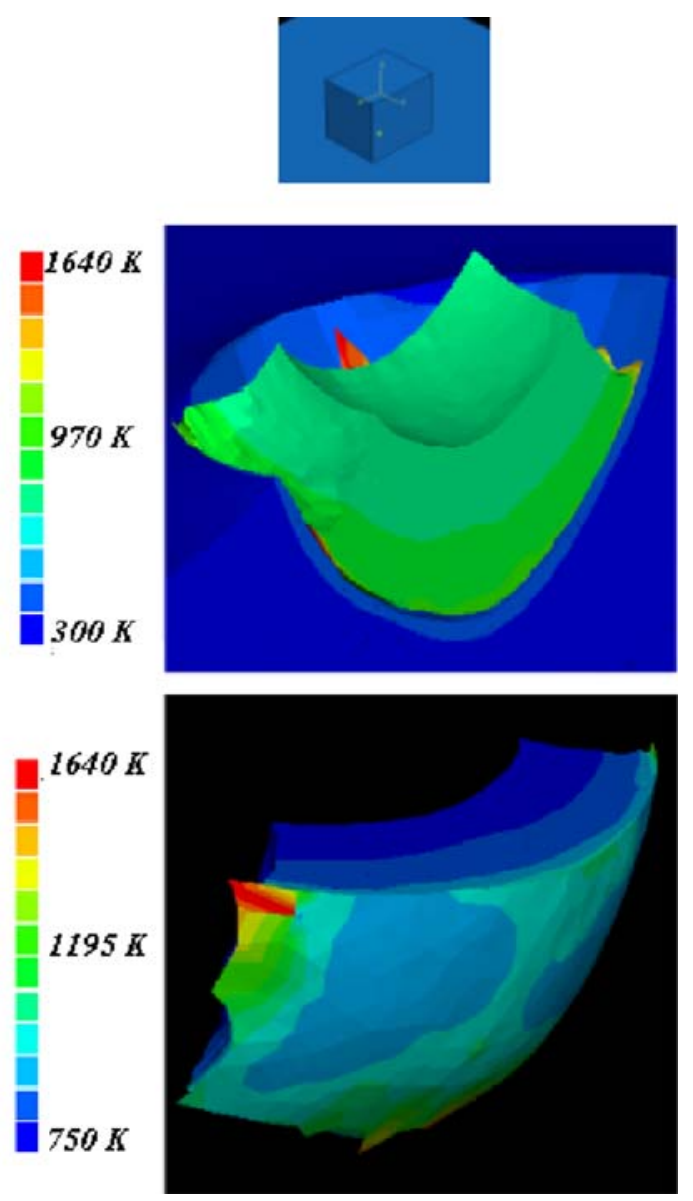

Fig. 10-Temperature contours of SF 0.8 particles with different orientations at impact (top: before impact; middle: after impact; and bottom: interfacial contact surface). 


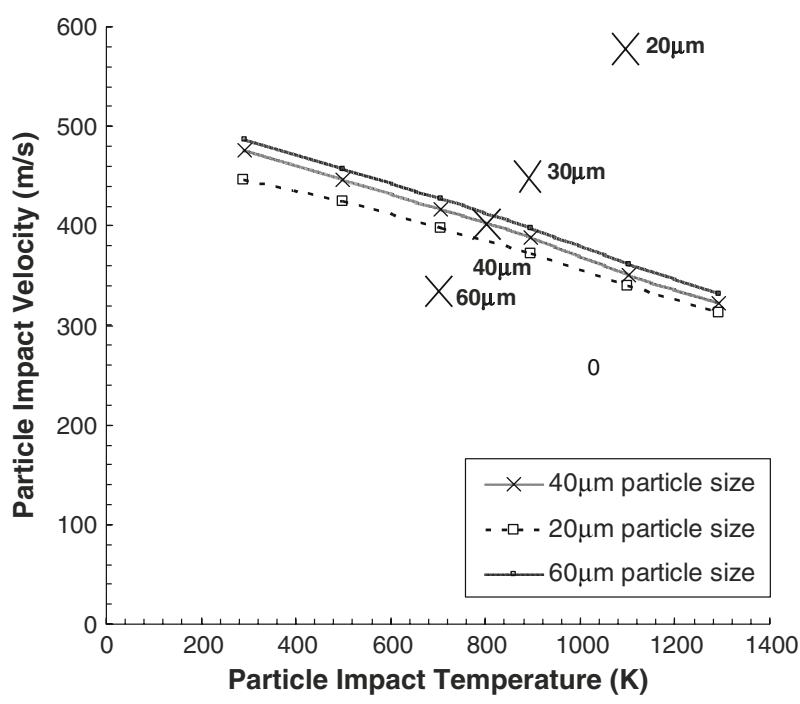

Fig. 11-Critical impact parameters for spherical particles.

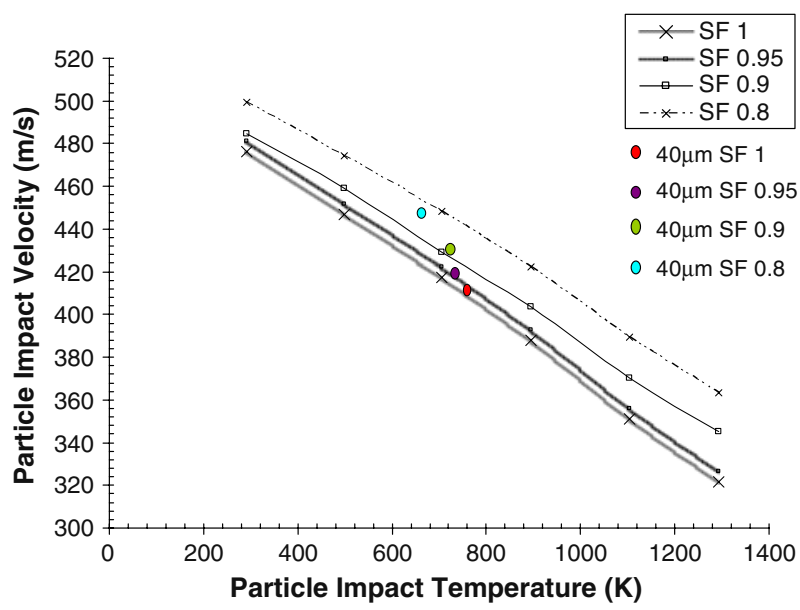

Fig. 12-Critical impact parameters for different shape $40-\mu \mathrm{m}$ particles.

The efficiency of deposition of nonspherical particles is illustrated with Figure 12 and shows the critical velocity profiles for $40-\mu \mathrm{m}$ particles. The color dots are the results taken from an in-flight particle simulation of an HVOF process. When the shape factor is reduced from 1 to 0.9 , the increase in drag due to the increased roughness of the particle surface increases its impact velocity, and while this slightly reduces its temperature, the impact properties are marginally improved, illustrated by the increasing distance between each colored point and its line of associated shape factor. However, for a powder particle with an SF of 0.8 , the increased momentum is counteracted by a decrease in particle temperature, which prohibits sufficient plastic deformation for adequate bonding.

When the particle size is reduced to $20 \mu \mathrm{m}$, as shown in Figure 13, the HVOF gun achieves a high level of momentum output for particles of all varied SF, and all exceed the critical impact requirements.

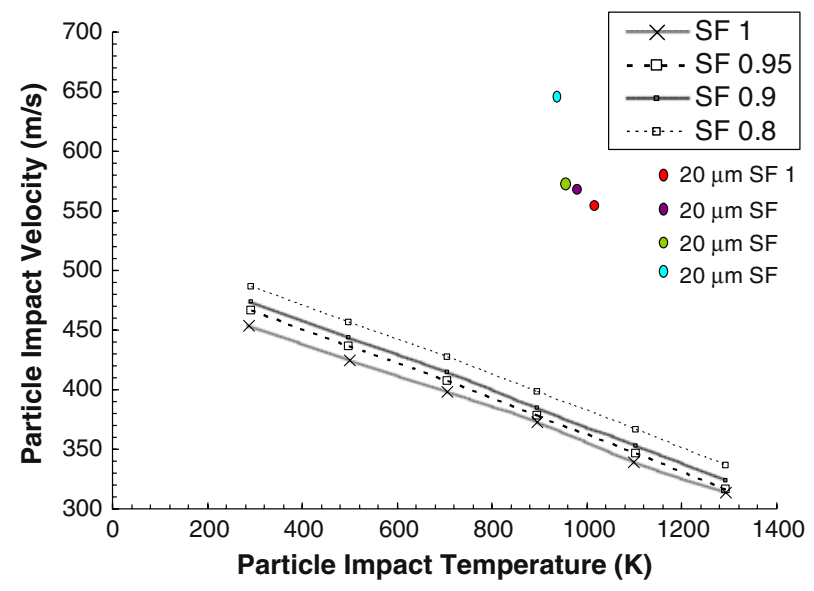

Fig. 13-Critical impact parameters for different shape $20-\mu \mathrm{m}$ particles.

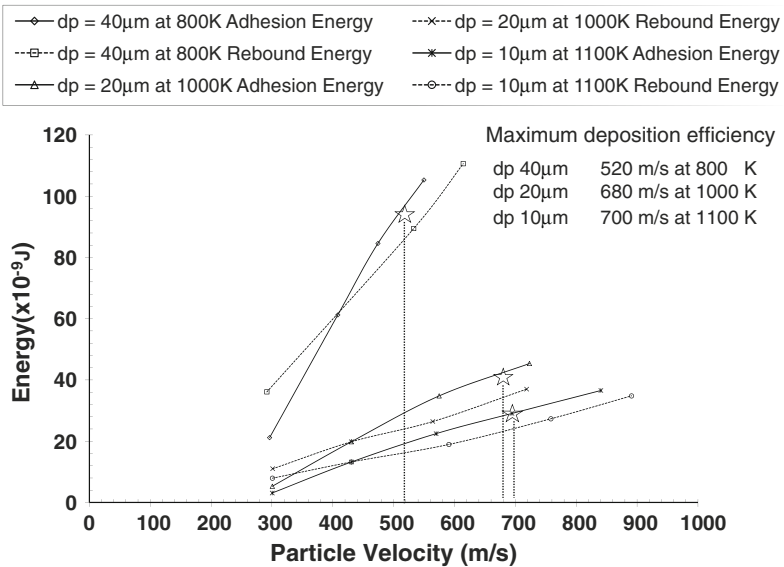

Fig. 14 Correlations for adhesion and rebound energies at different impact velocities for spherical particles.

Figures 11 through 13 are created according to the bonding criterion of adiabatic shear instability reported by Reference 19. An alternative method of finding critical impact velocities is by calculating the adhesion and rebound energies of the particle. A very good description and implementation of this method is provided by Reference 29. During impact, an elastic collision takes place, which is followed by an elastic unloading during which period the shape of the particle is recovered partially. The energy required to bounce the particle from the substrate during the unloading moment is defined as rebound energy. Adhesion energy is defined as the energy needed to detach the bonded particles from the substrate. The energy-based method is applied here to examine spherical powder for comparison with the former method. Figure 14 shows the way in which the two aforementioned energies change as a function of impact velocity. The particle is assumed to attach onto the substrate when the adhesion energy is higher than the rebound energy. For low velocity, the adhesion energy is lower than the rebound energy and 
the particle cannot be deposited. The impact velocity where the two curves intersect is the critical velocity. The optimum impact velocity for maximum deposition efficiency exists at the peak value of the difference between the adhesion energy and the rebound energy. The optimum impact velocities are listed in the top of Figure 14. The energy-based results are in good agreement with the previous calculation of critical velocity of about $450 \mathrm{~m} / \mathrm{s}$ for the $40-\mu \mathrm{m}$ spherical particle at a temperature of $730 \mathrm{~K}$. The results are also applicable to critical impact velocities for different particle sizes, and the energy curves that intersect at different velocities for different sizes of spherical particles are also shown in Figure 14. It should be noted that the critical condition in Figures 11 through 13 is the minimum requirement for bonding, which is the point of their intersection in Figure 14. The maximum deposition efficiency given in Figure 14 is where the largest difference between the adhesion energy and the rebound energy exists. The results for $40-\mu \mathrm{m}$ particles in both cases are similar; however, the energy-based method is only applicable to the spherical particle.

Experimental work has shown that an optimal impact velocity exists for thermal spray powder particles. ${ }^{[29]}$ While accelerating Al-Si particles uses the cold spray method, the particle impact velocity is measured using the SprayWatch system (Oseir Ltd., Tampere, Finland). The bonded particles are then examined visually using scanning electron microscopy, and the deposition efficiency is quantitatively assessed using energy dispersive spectroscopy. The effectivenss of the deposition is analyzed by calculating the adhesion and rebound energies. The experimental results demonstrate that an optimal impact velocity for maximum deposition rate exists, which is a result of competition between adhesion and rebound energies. Such a trend has been confirmed in this simulation of HVOF-sprayed particles; moreover, a similar magnitude $\left(10^{-9}\right)$ of adhesion and rebound energies is found.

\section{CONCLUSIONS}

An FEA model has been developed to study the impingement process of solid particles and the bonding mechanism between the particle and substrate. The results provide insights into the intricate interaction between plastic deformation and bonding formation. A summary of conclusions are as follows.

1. Due to thermal-softening effects, the particle is deformed to a greater extent than the substrate, resulting in a lower crater surface temperature compared to that of the particle interfacial region.

2. The kinetic energy of the particle prior to impact plays a dominant role in particle stress localization and melting of the interfacial region.

3. Nonspherical particles require more kinetic energy for good adhesion on the substrate. Forty-micron particles with $\mathrm{SF}<0.8$ sprayed by the HVOF gun are not able to form adequate bonding with the substrate.
4. The orientation of the nonspherical particle at impact affects the plastic deformation rate in the interfacial region and consequently the bond strength, which gives more complexity for spraying nonspherical powder.

It should be noted that the predicted higher temperature along the particle edges seems to contradict the results from the existing tin droplet models. ${ }^{[32,33]}$ The transfer of kinetic energy to internal energy is not significant in the case of low speed tin droplets. ${ }^{[32,33]}$ The edges of the splat make first contact with the lowtemperature substrate and are more exposed to the environment; therefore, the edges have low temperature and solidification is faster. For high speed solid particles in our case, the transfer of kinetic energy to internal energy is dominant. The edges have higher velocity, so more kinetic energy is transferred to internal energy to increase the temperature.

\section{ACKNOWLEDGMENTS}

The authors gratefully acknowledge the financial support from the UK DTI Technology Programme (Grant No. TP/J3019E) and EC FP7 Simuspray Project (Grant No. 230715).

\section{REFERENCES}

1. S. Gu, D.G. McCartney, C.N. Eastwick, and K.A. Simmons: J. Therm. Spray Technol., 2004, vol. 13, pp. 200-13.

2. S. Gu, C.N. Eastwick, K.A. Simmons, and D.G. McCartney: J. Therm. Spray Technol., 2001, vol. 10, pp. 461-69.

3. S. Kamnis and S. Gu: Chem. Eng. Sci., 2006, vol. 61, pp. 5427-39.

4. S. Kamnis and S. Gu: Chem. Eng. Proc., 2006, vol. 45, pp. 246-53.

5. N. Zeoli, S. Gu, and S. Kamnis: Comp. Chem. Eng., 2008, vol. 32, pp. 1661-68.

6. T.C. Hanson, C.M. Hackett, and G.S. Settles: J. Therm. Spray Technol., 2002, vol. 11, pp. 75-85.

7. M. Cherigui, Z. Salhi, N.E. Fenineche, P. Gougeon, and C. Coddet: Mater. Lett., 2005, vol. 59, pp. 463-67.

8. S. Kamnis, S. Gu, T.J. Lu, and C. Chen: Comp. Mater. Sci., 2008, vol. 43, pp. 1172-82.

9. S. Kamnis, S. Gu, and N. Zeoli: Surf. Coat. Technol., 2008, vol. 202, pp. 2715-24.

10. N. Zeoli, S. Gu, and S. Kamnis: Int. J. Heat Mass Transfer, 2008, vols. 15-16, pp. 4121-31.

11. S. Kamnis and S. Gu: J. Phys. D: Appl. Phys., 2005, vol. 38, pp. 3664-73.

12. S. Kamnis and S. Gu: J. Phys. D: Appl. Phys., 2008, vol. 41, p. 165,303 (7 pp.).

13. D.L. Gilmore, R.C. Dykhuizen, R.A. Neiser, T.J. Roemer, and M.F. Smith: J. Therm. Spray Technol., 1999, vol. 8, pp. 576-86.

14. C.J. Li and W.Y. Li: Surf. Coat. Technol., 2003, vol. 167, pp. 278 83.

15. T.H. Van Steenkiste, J.R. Smith, and R.E. Teets: Surf. Coat. Technol., 2002, vol. 154, pp. 237-52.

16. R.C. Dykhuizen, M.F. Smith, D.L. Gilmore, R.A. Neiser, X. Jiang, and S.J. Sampath: Therm. Spray Technol., 1999, vol. 8, pp. $559-67$.

17. M. Grujicic, J.R. Saylor, D.E. Beasley, W.S. DeRosset, and D. Helfritch: Appl. Surf. Sci., 2003, vol. 219, pp. 211-23.

18. M. Grujicic, C.L. Zhao, W.S. DeRosset, and D. Helfritch: Mater. Des., 2004, vol. 25, pp. 681-88.

19. H. Assadi, F. Gartner, T. Stoltenhoff, and H. Kreye: Acta Mater., 2003, vol. 51, pp. 4379-94. 
20. T.W. Wright: Int. J. Plast., 1992, vol. 8, pp. 583-602.

21. T.W. Wright: Mech. Mater., 1994, vol. 17, pp. 215-22.

22. D.Y. Tzou: ASME J. Heat Transfer, 1995, vol. 117, pp. 8-16.

23. G.S. Prakash, S.S. Reddy, S.K. Das, T. Sundararajan, and K.N. Seetharamu: Num. Heat Transfer A, 2000, vol. 38, pp. 513-32.

24. R. Kapoor and S. Nemat-Nasser: Mech. Mater., 1998, vol. 28, pp. $1-12$.

25. E. Aldie and Jr. Johnson: Technical Note 3309, National Advisory Committee for Aeronautics, Washington, DC, 1954.

26. G.R. Johnson and W.H. Cook: Proc. 7th Int. Symp. on Ballistics, The Hague, The Netherlands, 1983, pp. 541-48.

27. W. Kohlhofer and R.K. Penny: Int. J. Press. Ves. Pip., 1996, vol. 66, pp. 333-39.
28. R.B. Clough, S.C. Webb, and R.W. Armstrong: Mater. Sci. Eng. $A$, 2003, vol. 360 , pp. $396-407$

29. J. Wu, H. Fang, S. Yoon, H. Kim, and C. Lee: Scripta Mater., 2006, vol. 54, pp. 665-69.

30. Y.V. Kurochkin, Y.N. Demin, and S.I. Soldatenknov: Chem. Petrol. Eng., 2002, vol. 38, pp. 245-48.

31. B. Jodoin, L. Ajdelsztajn, E. Sansoucy, A. Zúñiga, P. Richer, and E.J. Lavernia: Surf. Coat Technol., 2006, vol. 201, pp. 3422-29.

32. V. Mehdi-Nejad, J. Mostaghimi, and S. Chandra: Int. J. Comput. Fluid Dyn., 2005, vol. 19, pp. 105-13.

33. R. Ghafouri-Azar, S. Shakeri, S. Chandra, and J. Mostaghimi: Int. J. Heat Mass Transfer, 2003, vol. 46, pp. 1395-1407. 